LAWRENCE LIVERMORE N A T IO N A L LABORATORY
Precision Manufacturing of Inertial

Confinement Fusion Double Shell Laser Targets for OMEGA

Robin L. Hibbard, Matthew J. Bono, Peter A. Amendt, Don W. Bennett, Carlos Castro

November 24, 2003

Fusion Science and Technology 
This document was prepared as an account of work sponsored by an agency of the United States Government. Neither the United States Government nor the University of California nor any of their employees, makes any warranty, express or implied, or assumes any legal liability or responsibility for the accuracy, completeness, or usefulness of any information, apparatus, product, or process disclosed, or represents that its use would not infringe privately owned rights. Reference herein to any specific commercial product, process, or service by trade name, trademark, manufacturer, or otherwise, does not necessarily constitute or imply its endorsement, recommendation, or favoring by the United States Government or the University of California. The views and opinions of authors expressed herein do not necessarily state or reflect those of the United States Government or the University of California, and shall not be used for advertising or product endorsement purposes. 


\title{
PRECISION MANUFACTURING OF INERTIAL CONFINEMENT FUSION DOUBLE SHELL LASER TARGETS FOR OMEGA
}

\author{
Robin L. Hibbard, Matthew J. Bono, Peter A. Amendt, Don W. Bennett, and Carlos Castro \\ Lawrence Livermore National Laboratory \\ P.O. Box 808, Mail Stop L-537, Livermore Ca, 94550 \\ hibbard2@llnl.gov
}

\begin{abstract}
Double shell targets have been built by Lawrence Livermore National Laboratory (LLNL) for inertial confinement fusion (ICF) experiments on the Omega laser at the University of Rochester and as a prelude to similar experiments on NIF. Of particular interest to ICF studies are high-precision double shell implosion targets for demonstrating thermonuclear ignition without the need for cryogenic preparation. Because the ignition tolerance to interface instabilities is rather low, the manufacturing requirements for smooth surface finishes and shell concentricity are particularly strict. This paper describes a deterministic approach to manufacturing and controlling error sources in each component. Included is the design philosophy of why certain manufacturing techniques were chosen to best reduce the errors within the target. The manufacturing plan developed for this effort created a deterministic process that, once proven, is repeatable. By taking this rigorous approach to controlling all error sources during the manufacture of each component and during assembly, we have achieved the overall $5 \mu \mathrm{m}$ dimensional requirement with sub-micron surface flaws. Strengths and weaknesses of the manufacturing process will be discussed.
\end{abstract}

\section{I . INTRODUCTION}

Lawrence Livermore National Laboratory (LLNL) manufactures laser targets for experiments on the Omega laser at the University of Rochester and is preparing to build targets for the National Ignition Facility (NIF). These targets serve university collaborations, high energy density physics studies, and inertial confinement fusion (ICF) customers. Of particular interest to ICF studies are highprecision double shell implosion targets for demonstrating thermonuclear ignition without the need for cryogenic preparation. The suite of double shell ignition designs for the $\mathrm{NIF}^{1}$ consists of a low-Z outer shell that absorbs laser generated $\mathrm{x}$-rays, implodes, and then collides with a smaller high- $Z$ inner shell containing the high-pressure deuteriumtritium fuel. Because the ignition tolerance to interface instabilities is rather low, the manufacturing requirements for smooth surface finishes and shell concentricity are particularly strict. As a prelude to fabricating and fielding ignition double shell targets on the NIF, an effort is underway on the Omega laser facility to build and field scaled ignition-like double shells.

An exploded view of the double shell target appears in Figure 1. The design consists of an inner plastic capsule with an outer diameter (OD) of $244 \mu \mathrm{m}$ and a wall thickness of $15 \mu \mathrm{m}$. After manufacturing the targets, the inner capsules are filled with deuterium gas at 50-65 atm prior to the laser shots. The inner capsule is suspended in two hemispherical shells (hemis) of low density CRF aerogel with a thickness of $220 \mu \mathrm{m}$. The outer ablator hemis have an outer diameter of $550 \mu \mathrm{m}$ and are $52 \mu \mathrm{m}$ thick. They are made of an LLNL fabricated $1 \%$ atomic weight bromine doped polystyrene and mate at a step joint. A bonded joint secures the two ablator hemis together and is designed to bond only in the outer half of the step joint, where a $2 \mu \mathrm{m}$ gap has been intentionally left for the adhesive to fill, as shown in Figure 2.

The following capsule parameters are particularly important to these implosion experiments: concentricity of

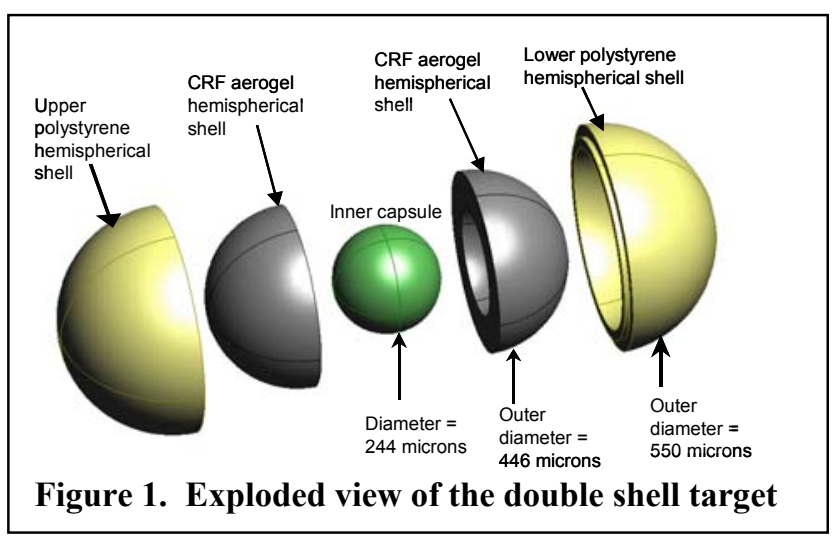


the two shells, thickness uniformity of the inner and outer shells, roughness of the various spherical surfaces, void volume fraction of the cyanoacrylate in the outer shell bond joint, and the pore size of the aerogel. All of these non-ideal effects can potentially degrade the performance of the target by causing hydrodynamic instabilities, so they require accurate pre-shot characterization. The overall requirements are to maintain the dimensional tolerance of each component to $\pm 1 \mu \mathrm{m}$ and all internal flaws to less than \pm 0.5 $\mu \mathrm{m}$. Surface roughness requirements for the polystyrene surfaces are $0.01 \mu \mathrm{m}$ RMS for Legendre mode numbers ten and greater. The inner capsule and outer shell are required to be concentric to within $5 \mu \mathrm{m}$. This specification on shell concentricity is important for constraining the degree of performance degradation from an asymmetric shell collision. Similarly, any flaws or non-uniformities in the outer shell or bond joint can cause deviations from a spherical implosion, leading to degraded performance.

The objectives of this paper are to show how LLNL's precision engineering team systematically approached the manufacture of a double shell target with a deterministic manufacturing plan and to discuss process limitations. An error budget was used to quantifiably study the manufacturing process and identify the effects of errors in each step. The discussion of the manufacturing steps will highlight how each component is built with known datums and reference surfaces, how these are maintained during the entire manufacturing process, and how the manufacturing plan created a deterministic, repeatable process. Included is the design philosophy of why certain manufacturing techniques were chosen to best reduce the errors within the target. By taking this rigorous approach to controlling all error sources during machining and assembly, one can attempt to achieve the requirements of $\pm 1 \mu \mathrm{m}$ dimensional accuracy, sub-micron surface flaws, and $5 \mu \mathrm{m}$ concentricity between the two shells. This contrasts with previous literature in the 1980's and the 1990's that described the manufacture of double shell targets using methods that introduce uncertainties into the manufacturing process, such as coating mandrels and releasing free-standing components, or backfilling materials to allow machining of components, then extracting the filler material and dealing with part shrinkage. ${ }^{2,3}$ The current effort was designed to involve a more deterministic manufacturing plan in order to avoid the uncertainties associated with these previous methods.

\section{MATERIAL ISSUES}

The inner capsules of the target are composed of plastic and are supplied by General Atomics (GA). The designed diameter of the capsules is $240 \pm 0.5 \mu \mathrm{m}$ with a wall thickness of $15 \mu \mathrm{m}$. The actual size of the capsules varies from 239 to $247 \mu \mathrm{m}$, and the uncertainty in the wall thickness is $\pm 0.8 \mu \mathrm{m}$. There is also a "p2" or ellipsoid variation of up to $1 \mu \mathrm{m}$. The capsules are measured by GA by taking three equatorial traces around the sphere using a spheremapper. $^{4}$ The capsule is supported inside the ablator shell by a $50 \mathrm{mg} / \mathrm{cc}$ carbonized resorcinol formaldehyde aerogel (CRF), with sub micron cell size. If appropriate cutting conditions are used, this material can be diamond turned with micron accuracy.

Polystyrene made at LLNL is given a bromine doping of $1 \%$ by atomic weight to shield the inner shell from 2-5 $\mathrm{keV} \times$ rays generated in the laser-irradiated high- $Z$ enclosure (hohlraum). The method used to manufacture this material is known to potentially introduce residual stresses that can cause crazing of the surface of the material after aging, exposure to UV light, or application of certain bonding agents. At the scale of $550 \mu \mathrm{m}$ OD with a wall thickness of $50 \mu \mathrm{m}$, any residual stresses, crazing, or cracking could affect the dimensional consistency of the components. Initial attempts to fabricate ablator components from this material revealed that the machined

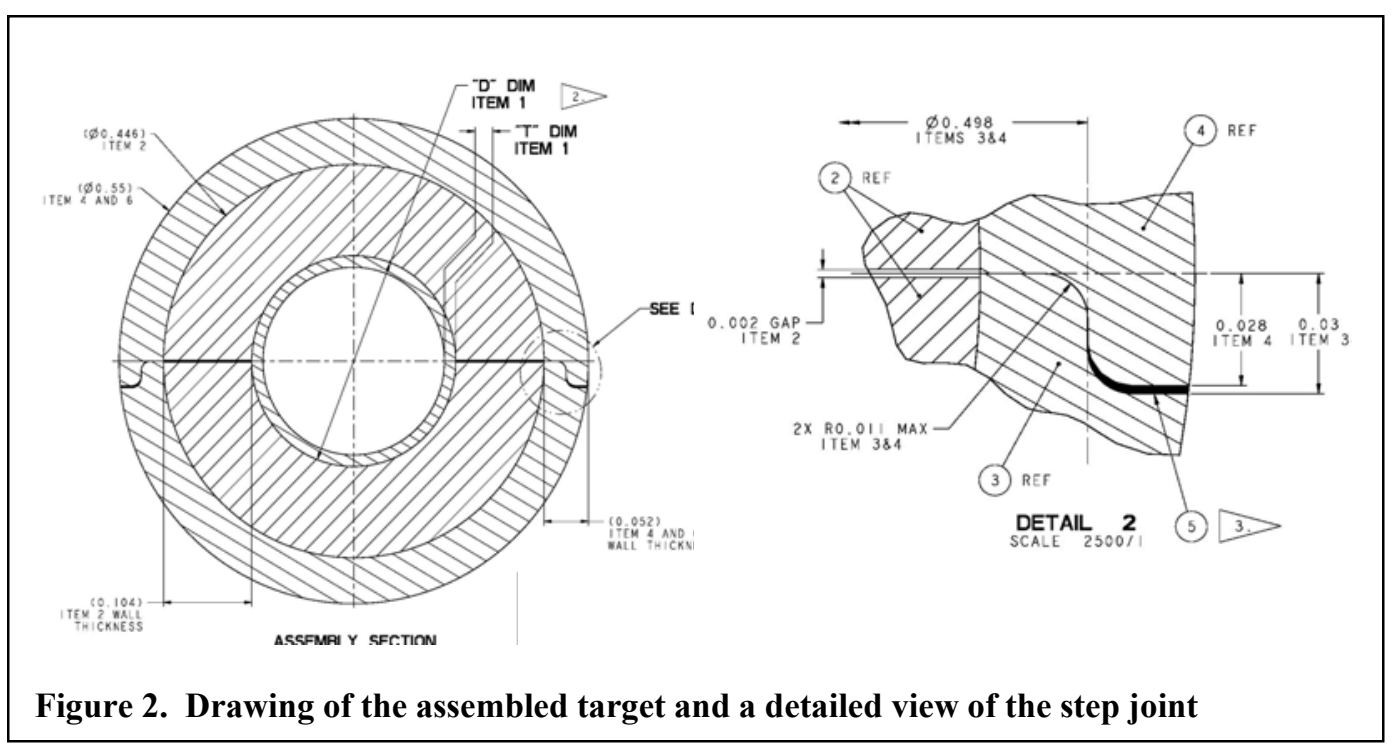

surfaces would craze and develop cracks after several days. The application of cyanoacrylate to the surfaces of this material caused immediate crazing and cracking. In an effort to relieve the residual stresses in the material prior to machining, the material was heat treated at $135^{\circ} \mathrm{C}$ for 24 hours. This heat treated material did not experience problems with crazing, even after several months of storage in a desiccant jar that was shielded from UV light, and it proved to be 
usable for the manufacture of the targets. However, a rigorous annealing process has yet to be identified.

Both a cyanoacrylate and an epoxy are used in the manufacturing process. The designed bond joint requires that the density of the adhesive match that of the polystyrene shell to within $10 \%$. The adhesive must wick into the $2 \mu \mathrm{m}$ gap in the joint and completely fill it. Otherwise the resulting voids in the joint would adversely affect the target. The adhesive inside the gap cannot contain impurities or binders with a high $\mathrm{Z}$ that obscure X-rays. Furthermore, the adhesive must not wick past the inner step in the joint and into the CRF, because the additional full density material between the shells would degrade the performance of the target. The adhesive must also be able to cure without affecting the dimensional stability of the target.

A transportation container with a single target mounted inside is pressurized at either 50 or $65 \mathrm{~atm}$ of $\mathrm{D}_{2}$ gas depending on physics specification. During this process the target is diffusion filled through the outer plastic shell, the intermediate shell of porous aerogel foam and into the inner capsule. The target is stored in this pressurized container until 30-60 minutes prior to the shot, and then it is removed for insertion into the target chamber. To remove a target, the container is connected to a source at the same pressure to verify the pressure in the transportation container. Then a valve is opened to allow depressurization in 10-15 minutes at near constant rate. Since the plastic shell and the aerogel foam have a much higher permeation rate than the inner capsule, these two components essentially come to ambient pressure fairly quickly. However, the inner capsule can remain at filled pressure far exceeding the time required to execute the shot. As the depressurization valve is opened to the source, a pressure drop would signify that the container is at a lower pressure - indicating a leak in either the target or the container. No such abnormality was noted in the entire batch of targets. As an integral part of developing a viable target construction process, laboratory tests that followed the expected pressurization/ depressurization timing sequence were conducted. This was done to verify that the outer shell adhesive joint would survive a transient differential pressure across the shell wall. Test results indicated that the bond joint could withstand more than four times the fill pressure.

\section{MACHINING OF THE COMPONENTS}

To fabricate each target, the CRF hemis are machined, the inner contours of the ablator hemis and the step joint are machined, the five components are assembled and bonded, and then the outer contour of the target is machined. The following discussion of the machining processes will follow this sequence. This sequence of manufacturing steps differs from recent efforts at LLNL to manufacture larger double shell targets, in which freestanding ablator hemis were placed around the CRF hemis and inner capsule and then bonded together. ${ }^{5}$ This new manufacturing method has many advantages, including the ease of the bonding process, less precision is required in that an excessive amount of adhesive can be applied and then later machined off, this improves the fit and finish of the outer contours, and it improves the precision of design by not having to transfer and handle individual free standing hemis and position them in a vacuum chuck. The design philosophy behind this manufacturing plan is to know the reference position and size of each component throughout the manufacturing process and to minimize points where dimensional uncertainty can affect the quality of the target. This systematic approach can be very time consuming, but when done properly, it produces a repeatable process that allows subsequent builds to be efficient.

The parts are machined on a Precitech Nanoform 200 diamond turning machine (DTM) equipped with a B-axis, using a single point diamond tool with a nose radius of 5 $\mu \mathrm{m}$. During all DTM processes, precision holders that locate onto a checking ball allow the parts to come on and off the machine with a positional repeatability of $\pm 0.25 \mu \mathrm{m}$. Setting the tool position on the DTM is done optically and initially orients the tool to within approximately $\pm 1.5 \mu \mathrm{m}$. The tool set is then refined using a manual approach that is time-consuming but sets the tool position with an accuracy of $\pm 0.25 \mu \mathrm{m}$ in each direction. When machining the inner contours of the CRF and ablator hemis, the tool must be kept normal to the contour, so that adequate clearance is maintained between the clearance faces of the tool and the edge of the machined contour. All machining is performed with a spindle speed of $1700 \mathrm{rpm}$ and a depth of cut of 3.5 $\mu \mathrm{m}$ for roughing cuts and $1.25 \mu \mathrm{m}$ for finishing cuts. The feedrate of the operations varies, because the Precitech controller does not maintain a constant feedrate when moving the B-axis simultaneously with another axis, but the feedrate for finishing cuts is generally around 0.12 microns/revolution.

\section{III.A. Vacuum Chucks}

Vacuum chucks are used in three places during this target build. One vacuum chuck is used to hold the OD of the CRF hemis while machining the inner contours, and another vacuum chuck is used to hold the OD of the bonded target while machining the final portion of the outer contour of the ablator. Another flat vacuum chuck is used to transfer the CRF pieces during assembly. The manufacturing method is similar for each of the three chucks and is illustrated in Figure 3.

To create the vacuum chuck that holds the CRF, a hole is drilled in a brass rod with a polystyrene disk on the end, which is mounted on a precision holder that allows vacuum to be pulled through the spindle of the machine, (Figure 3a). Then a hemispherical contour with a diameter slightly 
smaller than the OD of the CRF hemis is machined into the polystyrene disk, and a set of concentric channels $97 \mu \mathrm{m}$ wide and $15 \mu \mathrm{m}$ deep is cut into the inner contour (Figure $3 b)$. Then a groove is scratched into the contour to connect each of the channels (Figure 3c). The part is subsequently placed back in the DTM, and the contour is machined to its final diameter of $446 \mu \mathrm{m}$ to match the OD of the CRF hemis (Figure 3d). $\pm 0.25 \mu \mathrm{m}$, which is the minimum manual jog size of the DTM. This touch-off process allows the position of the part to be reestablished. The sphere is then faced off to create a solid hemisphere, and the internal contour is machined into the CRF to complete the hemi (Figure 4c). Each internal contour is custom fit to a particular capsule size. The length of the excess neck material is minimized to reduce the torsional moment on the vacuum chuck imposed by the tool cutting forces. When complete, the parts are inspected with

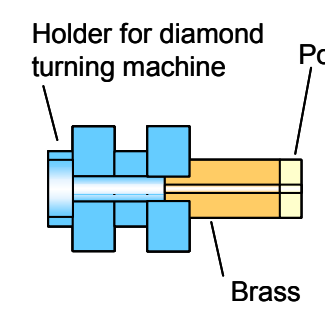

a)

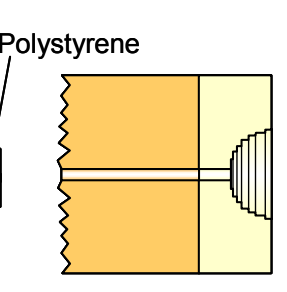

b)

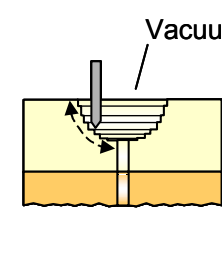

c)



d)
Figure 3. Procedure for manufacturing a vacuum chuck a microscope to measure the ID. The uncertainty in this measurement is $\pm 1 \mu \mathrm{m}$.

This machining process is deterministic and produces dimensionally stable CRF components. Note that previous efforts to build low density support structures for the inner capsule used casting or filling of foam with support material that had to be leached out, which caused linear shrinkage of up to $2 \%$. $^{3}$

\section{III.B. CRF Hemispheres}

Figure 4 illustrates the process for machining each CRF hemi. First, a large piece of the CRF is glued to an arbor mounted to a precision holding fixture. The CRF is then turned down to create a partial sphere of diameter $446 \mu \mathrm{m}$, supported by a neck of material, which will be the outer contour of the hemi (Figure 4a). After measuring the OD of the sphere with a microscope, the sphere is manually broken off at the neck using a surgical blade, picked up using a vacuum pencil, and placed into the specially prepared vacuum chuck described above (Figure 4b).

\section{III.C. Polystyrene Ablator Components}

The ablator hemis are machined in two different stages. In the first stage, the inner contour and corresponding step joint are machined, as shown in Figure 5. The second stage is the machining of the outer contour of the ablators, but this second stage is performed after the target has been assembled and bonded and will be discussed in Section V. This sequence for building the targets has simplified the manufacturing process and improved the quality of the target as compared to LLNL's previous double shell target design. ${ }^{5}$

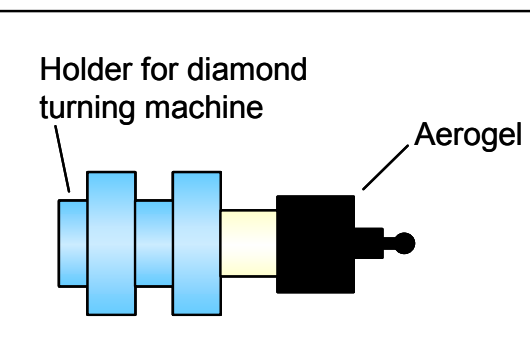

a)

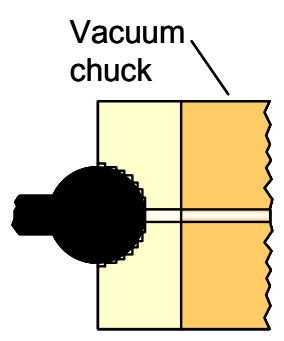

b)

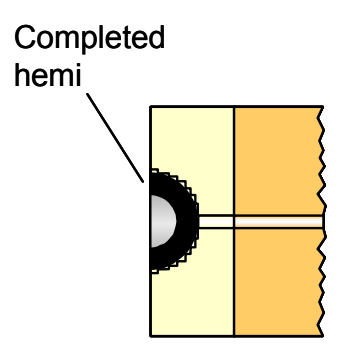

c)

To create each ablator component, the polystyrene material is mounted in a holder (Figure 5a), and a rough diameter and reference surface are cut (Figure 5b). This reference surface will be used in a subsequent process to establish a workpiece coordinate system when machining the outer contour of the ablators. The joint is also machined into the ablator component, along with a taper on the outside of the component that will facilitate bonding the ablator components after assembly.

Throughout the machining of the CRF, its position is deterministically known with respect to the DTM by orienting the hemis using vacuum chucks with reference surfaces. The only occasion in which the orientation of the part is not controlled with respect to a reference datum is when it is transferred to the vacuum chuck. To relocate the part once it is in the vacuum chuck, the tool is touched off on the face of the vacuum chuck, which is a known reference datum that can be located with an accuracy of

\section{ASSEMBLY AND BONDING}

An assembly fixture that uses the same workpiece holders that are used on the diamond turning machines is used for assembly. The assembly fixture uses an air bearing slide coupled in series with a spring and a force transducer to provide controlled compliance and control over the 

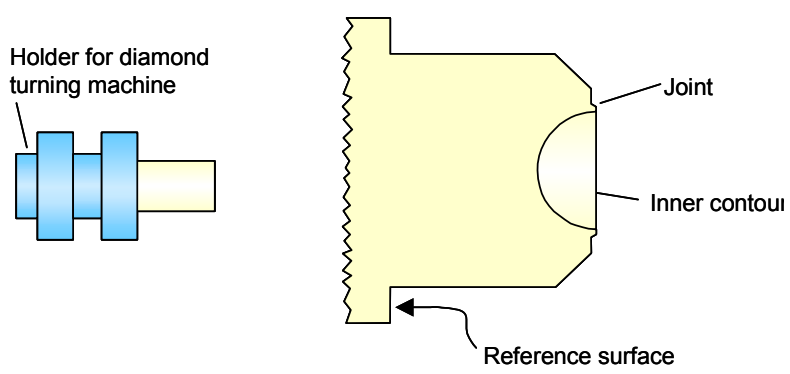

a)

b)

Figure 5. Procedure for machining the inner contours of the ablator components

assembly forces to within 1 gram, in order to avoid crushing the fragile components. During assembly, a high-resolution camera and a surgical microscope provide two orthogonal views of the mating components. Cleanliness and static electricity are important issues during assembly, because any dust or debris as small as $1 \mu \mathrm{m}$ can be large enough to cause ill-fitting pieces.

The assembly process is illustrated in Figure 6 . Initially, the lower ablator component is inserted into the lower actuator of the assembly station (Figure 6a). Then one of the CRF hemis is placed into the lower ablator component (Figure 6b). To perform this operation, the CRF hemi must be removed from its vacuum chuck by a transfer chuck and then lowered into the inner contour of the lower ablator component. Because the equator of the hemi is flush with the surface of the vacuum chuck, the part can be located axially by bottoming out the face of the vacuum chuck on the inner step of the joint on the lower ablator component. Note that the CRF hemi is maintained in a known angular orientation in each transfer operation, because it is handled only by precision transfer chucks that can pick up and transfer a component with $0.5 \mu \mathrm{m}$ repeatability.

The inner capsule is then placed on the platform of the assembly fixture, where it is picked up by the transfer chuck and inserted into the inner contour of the CRF hemi (Figure 6c). The upper CRF hemi is then taken directly from the

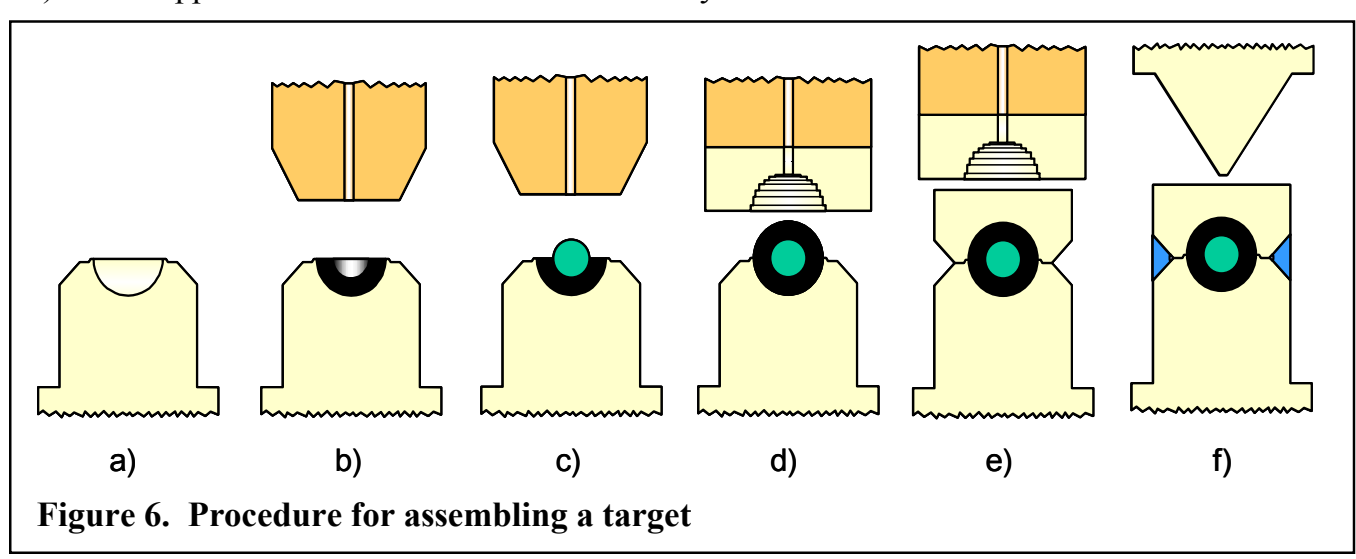

vacuum chuck in which it was machined and placed on top of the inner capsule (Figure 6d). The inner contours of each CRF hemi are machined to match the outer diameter of the corresponding capsule, but due to the uncertainty in the OD of the capsule, the potential exists for this step of the assembly process to cause a significant error in concentricity of the capsule with respect to the ablator components. The capsule could be loose inside the CRF hemis, or it could be larger than the machined cavities, which would cause at least one of the fragile CRF hemis to be crushed and distorted. In the final assembly step, the upper ablator component is placed over the CRF (Figure 6e) and mated with the joint in the lower component. The two ablator components are pressed together with a force of 10 grams, and the seating of the joint is visually inspected to ensure it has a uniform gap around the perimeter.

The bonding of the ablator components is done on two steps. First, cyanoacrylate is applied around the perimeter of the joint by placing a droplet on the end of a camel hair and running it around the perimeter. The volume of cyanoacrylate applied is sufficient to fill the $2 \mu \mathrm{m}$ gap in the joint, but it fills only the apex of the tapered sections. The joint has a straight step, and the inside surfaces of the joint are flush to prevent the adhesive from wicking into the CRF. Then the tapered sections of the components are filled with epoxy (Figure 6f), which is allowed to cure for 24 hours. This epoxy supports the joint during machining and is fully machined away in subsequent steps. By bonding the components prior to machining the outer contour, the need for precision dispensing of adhesives is avoided. Furthermore, crazing in the ablator material is not a concern for these targets. By machining the external surface after bonding the components, any crazing that occurs upon application of the cyanoacrylate is removed in subsequent machining, leaving a diamond turned quality surface.

\section{FINAL MACHINING}

There are three steps to the final machining: setting the tool, machining the first half of the outer contour and then transferring the target to a vacuum chuck, and machining the second half of the outer contour. The tool setting is critical for this operation, because any error in its position will cause wall thickness errors in the target. Therefore, before machining the outer contours of the ablators, the tool is reset using the procedure described earlier.

Figure 7 illustrates the procedure for machining the outer contour of the target. 


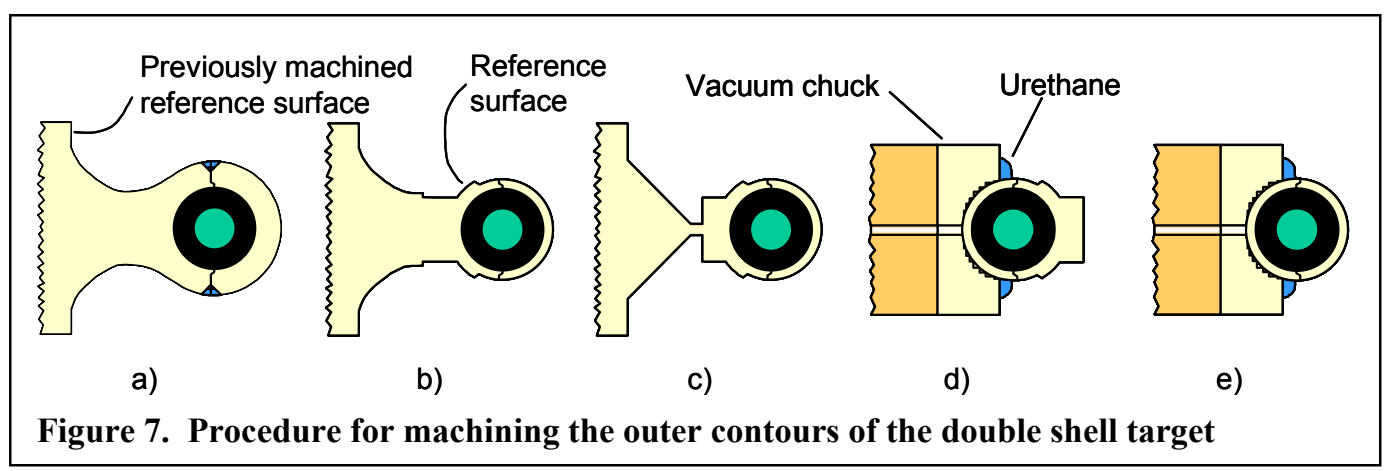

of the two hemispheres appears uniform on all targets. The area in which the two outer ablator surfaces were blended together is inspected with a WYKO NT8000 scanning white light interferometer to quantify the characteristics of the blend line, and the blended area appears to contain a surface flaw in the form of a step estimated at

The tool is touched to the previously machined reference surface on the lower ablator component to establish a workpiece coordinate system. Then the bonded assembly is machined from the form shown in Figure $6 \mathrm{f}$ to the form shown in Figure 7a. In this machining process, the tool also machines the OD of the shank near the reference surface. This machined OD serves as a reference diameter that can be measured with an uncertainty of \pm 1 um. Thus, by measuring this reference diameter, the OD at the equator of the machined target can be inferred.

Note that at this stage, the support shank diameter and length have been maintained to provide support, so the part will not deflect under the cutting forces. The epoxy inside the taper provides support to the bonded joint during machining. The free end is then machined to its final spherical form, and it includes another reference surface near the stem, as illustrated in Figure $7 \mathrm{~b}$. At this stage, the majority of the outer contour is complete. All of the epoxy has been machined away, and the joint is held together only by the cyanoacrylate in the $2 \mu \mathrm{m}$ gap. The stem holding the partially machined sphere is then reduced in size to a thin neck (Figure 7c), which is then broken off with a surgical blade. The free spherical end of the target is then gripped by the specially crafted vacuum chuck (Figure $7 d$ ), which holds the part while the remainder of the outer ablator surface is machined. To augment the grip of the vacuum chuck, a bead of urethane adhesive is applied to bond the part to the chuck. The remainder of the outer surface of the target is then machined (Figure 7e), and the final finishing pass blends together the surfaces on the outer contour. The urethane is then peeled off so that the completed target can be removed from the vacuum chuck.

\section{CHARACTERIZATION}

A completed target is shown in Figure 8. Once the target is completed, only external dimensional metrology and characterization can be performed. Visual inspection of the joint using an optical microscope indicates that the cyanoacrylate has fully wicked around the joint, and there are no visible voids or bubbles in the $2 \mu \mathrm{m}$ gap. The seating less than $0.3 \mu \mathrm{m}$ on all targets (radial perturbation $<0.1 \%$ ).

One of the most important characteristics of the targets is the concentricity between the ablator shells and the inner capsules, which is determined from contact radiographs of the completed targets. The contact radiographs have an estimated resolution of approximately $1 \mu \mathrm{m}$. Two orthogonal radiographs are taken of each target. Example radiographs for one of the targets appear in Figure 9.

For each target, two different fitting routines are used to calculate the concentricity errors in the plane of the joint and in the pole-to-pole direction. The six targets have concentricity errors of $1,3,4,4,5$, and $5 \mu \mathrm{m}$, which represent a $\Delta \mathrm{r} / \mathrm{r}$ of between $0.4 \%$ and $2 \%$.

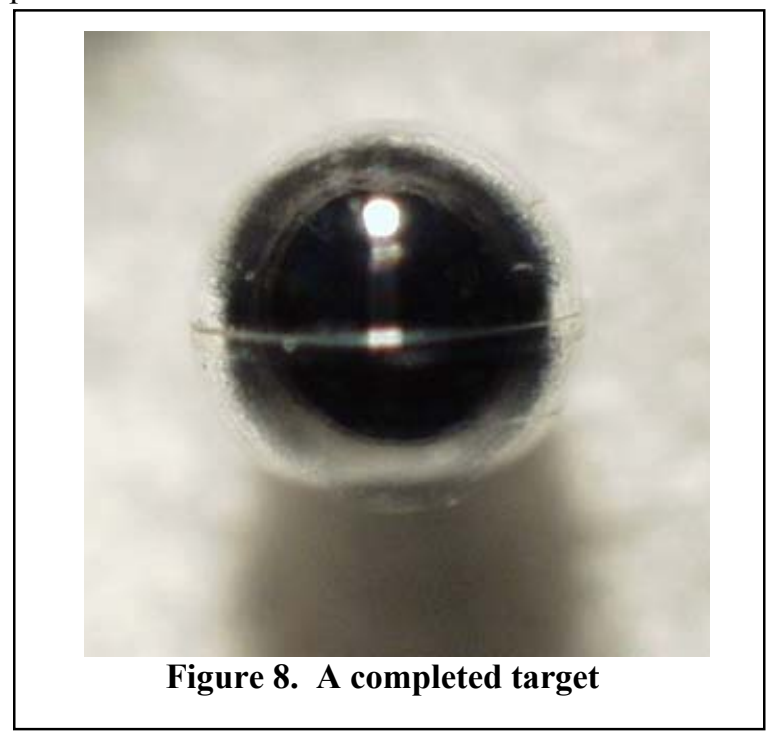

\section{SUMMARY}

By taking a rigorous approach to controlling the manufacturing process, double shell targets have been manufactured to meet the specifications of $\pm 1 \mu \mathrm{m}$ dimensional accuracy and $5 \mu \mathrm{m}$ concentricity. These targets provide physicists with an experimental target that may yield improved performance. This approach of using known manufacturing methods and pushing the limits of 


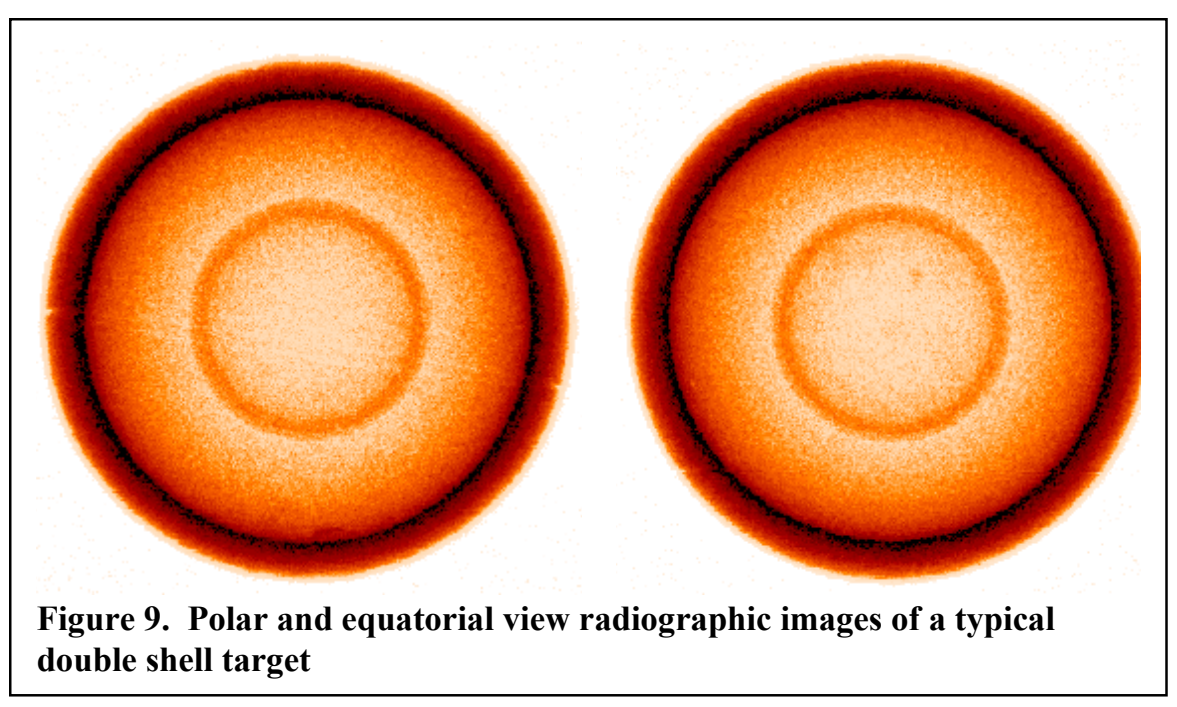

\section{ACKNOWLEDGEMENTS}

This work was performed under the auspices of the U.S. department of energy, by Lawrence Livermore National Laboratory, under contract W-7405-ENG-4.

\section{REFERENCES}

conventional processes can achieve high quality parts, because the manufacturing process has minimized the introduction of geometrical/dimensional uncertainties in each component.

The reality is that process control and development are the major tasks in executing a job like this. Most of the effort involves paying extreme care to the minute details at each step.

Throughout this process, we have identified areas where improvements can take place, and the preliminary error budget has been refined to identify the most important sources of error and uncertainty in the manufacturing process and to reveal their impacts on the quality of the completed targets. The error budget reveals that the key areas are developing a better assembly station, reducing the uncertainty in the measured diameters of the inner capsules, obtaining better metrology tools, and obtaining a better tool set station.
[1] P. A. AMENDT, et al., "Indirect-Drive Noncryogenic Double-Shell Ignition Targets for the National Ignition Facility: Design and Analysis", Phys. Plasmas 9, 2221 (2002).

[2] C. W. HATCHER, et. al., "Double-Shell Inertial Confinement Fusion Target Fabrication", J. Vac. Sci. Technol,. 18, 1187 (1981).

[3] J. R. DUKE, N. E. ELLIOT, et. al., "The Fabrication of Double Shell Targets for NOVA", Fusion Technol., 35, 90, (1999).

[4] R. L. McEACHERN, C. E. MOORE, R. J. WALLACE, "Design, Performance, and Application of an Atomic Force Microscope-Based Profilometer" J. Vac. Sci. Technol. A, 13, 983 (1995).

[5] M. J. BONO, R. L. HIBBARD, P. J. DAVIS, "Fabrication of the Double Shell 2 Laser Targets Via the Bonding of Hemispheres", Am. Soc. Prec. Eng., 30, 527 (2003). 